\title{
THEORY OF THE ROOT LINES OF THE GENERAL POLYNOMIAL
}

\author{
Nicholas Schmidt, Dipl. Ing. (graduated at the Institutul Polytehnic Timisoara, Romania) E-mail:
} nicholas.schmidt007@gmail.com

This paper describes the main characteristics of the root lines of polynomials with complex coefficients. Root lines are defined as the geometric loci of the roots of the real, respective imaginary part of the polynomial. A graphical representation of these lines offers a clear picture of the positions of the polynomial's roots. The coordinates of various points of the root lines can be obtained by use of a computer program. Each root line leads to a given root, this way the polynomial can be solved and graphically represented.

A short description of the rules of variation and properties of these root lines follows depending on the position of the roots of the polynomial. This study contains many numerical examples which show not only how the root lines, but also the polynomials with complex roots and coefficients work, how they can be studied, transformed and solved.

\section{A. Introduction}

This study is a continuation and completion of a study about the root lines presented at the 27-th ARA Congress in Oradea, Romania, May 29 - June 2, 2002.

The length of the study was then restricted to 5 pages, therefore some observations in the study could not be mathematically proved or demonstrated.

This paper presents all those observations and new explanations regarding the properties of root lines.

\section{B. Importance of the root lines.}

Root lines are characteristic for polynomials with complex roots. A polynomial of degree $n$ has $n$ roots and all roots can be complex. According to some scientific works the concept of complex roots has a particular importance to the physical and engineering sciences.

See Reference [4] Volume II, for the list of such cases.

The same [4], on page 413 and ff. also presents some curves which are root lines, but are called by the author $\mu(x, y)=c 1$ and $v(\mathrm{x}, \mathrm{y})=\mathrm{c} 2$. ( $\mu$ and $v$ are obviously the real and imaginary part of the polynomial).

The name "root lines" of this theory is given by me, and probably is not found in any other book or article.

In the same chapter of that book [4] is also mentioned that the angles between these curves at a multiple root are equal. It explains this with the vector dot product of the gradients. This study gives a more simple explanation for this. The studies mentioned in [4] are also restricted to polynomials with real coefficients.

My studies have no such restriction, because I found that they can easily be extended to cases with complex coefficients, so they are more general, without restriction.

\section{The basic form of a polynomial is}

$$
\mathrm{P}_{\mathrm{n}}(\mathrm{w})=\mathrm{C}_{\mathrm{n}} \mathrm{w}^{\mathrm{n}}+\mathrm{C}_{\mathrm{n}-1} \mathrm{w}^{\mathrm{n}-1}++\mathrm{C}_{1} \mathrm{x}+\mathrm{C}_{0}
$$

$\mathrm{w}$ is the independent variable of the polynomial which can be a real or a complex number; regarding w see also Par. I. The coefficients $C_{n}$ to $C_{0}$ are complex or real numbers.

\section{Roots of a polynomial.}

Roots (or zeros) of a polynomial are those values of the variable w for which the polynomial's value (both the real and the imaginary part) reduces to zero.

\section{E. Real roots of a polynomial.}

If the variable $w$ in relation (1) is a real number, and $\mathrm{P}(\mathrm{w})$ is represented along a straight reference line, then the roots are points where the curve crosses this line.

The number of real roots sometimes is equal with $n$, but in other cases less than $n$ (order of polynomial), even 0 .

\section{F. Complex roots of a polynomial.}

If we compare polynomial $x^{2}-9$ with $x^{2}+9$ then the condition $P=x^{2}-9=0$ can be written also $x^{2}=9$ and hence $\mathrm{x}=+3$ or $\mathrm{x}=-3$, so this polynomial has two real roots where the curve intersects the Ox line.

The polynomial $P=x^{2}+9$ on the other hand, as we can see from Fig. 1 has the minimum value for $\mathrm{x}=0$, then $\mathrm{P}=9$,

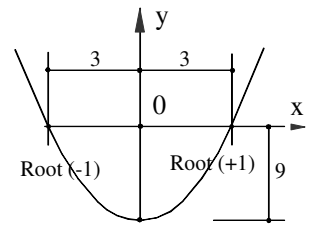

Polynomial $x^{2}-1$

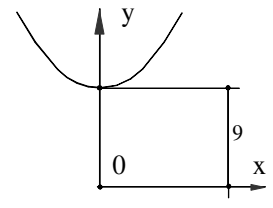

Polynomial $x^{2}+1$
Fig. 1

but it never reduces to zero.

From $\mathrm{P}=\mathrm{x}^{2}+9=0$ results $\mathrm{x}^{2}=-9$ or $\mathrm{x}=\sqrt{-9}=$ $3 \sqrt{-1}$

Because it is not possible to extract radical of -1 , this number is called imaginary unit.

So we reach to the notion of complex numbers which have the form $\mathrm{a}+\mathrm{bi}$ where $\mathrm{a}$ and $\mathrm{b}$ are real numbers and $\mathrm{i}=$ $\sqrt{-1}$ is the imaginary unit.

Then the number of roots of a polynomial is completely solved: Any polynomial has n roots which are either real or complex. Actually the real roots can be considered as complex numbers with $\mathrm{b}=0$.

\section{G. The second basic form of a polynomial.}

If all roots of a polynomial are known, then the polynomial can be written in another basic form 


$$
\mathrm{P}_{\mathrm{n}}(\mathrm{w})=\left(\mathrm{w}-\mathrm{w}_{1}\right)\left(\mathrm{w}-\mathrm{w}_{2}\right)\left(\mathrm{w}-\mathrm{w}_{\mathrm{n}}\right)
$$

where $\mathrm{w}_{1}, \mathrm{w}_{2}, \quad \mathrm{w}_{\mathrm{n}}$ are complex numbers and the polynomial in form (2) obviously reduces to zero if $\mathrm{w}$ is equal with one of the roots.

If the mathematical operations are performed, then (2) reduces to (1) because all operations are unique.

\section{H. Independence of the roots and root lines from the reference} axes.

This rule is very important for the study of the root lines. In relation (2), if we choose any point $\mathrm{M}\left(\mathrm{x}_{\mathrm{p}}, \mathrm{y}_{\mathrm{p}}\right)$ and calculate the value of the polynomial for this point $\mathrm{M}$, then we choose other two refernce axes $(\mathrm{Ox}, \mathrm{Oy})$, the value of the polynomial in the same point $M$ will be the same.

Proof.

In relation (2) both $\mathrm{w}$ and $\mathrm{w}_{1}, \mathrm{w}_{2}, \ldots \mathrm{w}_{\mathrm{n}}$ are measured from the same reference axes (Ox, Oy). If an axis is moved for ex. by $d$ then both $\mathrm{w}$ and $\mathrm{w}_{\mathrm{i}}$ increase by the same value $d$ and their difference remains the same.

This rule can be automatically extended to the root lines. A root line constructed in one system (of Ox, Oy) axes remains the same if the axes are moved because each point of the root line is a root. On the other hand coefficients of a polynomial change if the reference axes are moved.

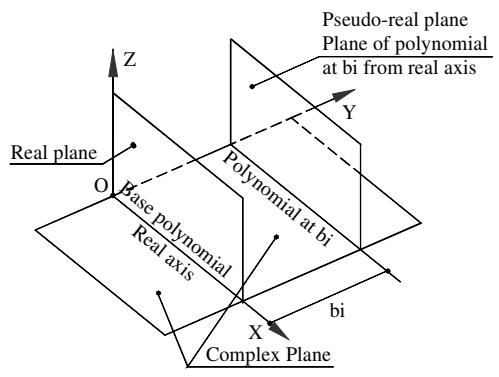

Fig. 2

\section{Representation of the roots and root lines of a polynomial.}

The real roots of a polynomial are represented along the Ox axis. The imaginary component bi of a complex root has a direction perpendicular to the real axis. So a complex number $\mathrm{a}+\mathrm{bi}$ is a vector whose real component $a$ has direction of the Ox axis and its imaginary component direction of the Oy axis. The complex variable $\mathrm{w}=\mathrm{a}+\mathrm{bi}=$ $\mathrm{x}+\mathrm{yi}$ then defines a complex plane (See Fig 2)'

Observation.

Fig. 2 has a tri-dimensional representation. The complex variable having two dimensions $(x+y i)$, it is natural to assume that the function $\mathrm{P}(\mathrm{x}+\mathrm{yi})=\mathrm{u}+\mathrm{vi}$ has also two components $\mathrm{u}$ (real part) and $\mathrm{v}$ (imaginary part) which are both represented in a direction $z$ perpendicular to the Oxy plane, so both $\mathrm{u}$ and $\mathrm{v}$ can be represented as two surfaces, each point of which has a value in direction $\mathrm{z}$. Therefore the complex variable will be noted in this study as $\mathrm{w}=\mathrm{x}+\mathrm{yi}$.
(Many authors use the notation $\mathrm{z}=\mathrm{x}+\mathrm{yi}$, but they don't have a figure like Fig. 2 in this paper).

In this study the letter $\mathrm{z}$ will be used for a variable whose direction is vertical and perpendiculat to the complex plane Oxy. Both u and v have direction of $\mathrm{z}$, but different values.

J. Points of root lines can be obtained by substitution of the complex variable in the polynomial.

If we substitute the complex variable $\mathrm{w}=\mathrm{a}+\mathrm{bi}$ in $\mathrm{a}$ polynomial $\mathrm{P}_{\mathrm{n}}$, then in the same point of the complex plane we will obtain two values: $u$ an vi. These values $u$ and $v$ are assumed to be vertical, i.e. perpendicular to the complex plane Oxy in the direction of the $\mathrm{Oz}$ axis (See Fig. 3)

The imaginary unit i only very rarely disappears. Therefore all terms multiplied by $i$ added up give an imaginary part noted generally with $v$. The other terms (without i) give the real part noted $u$.

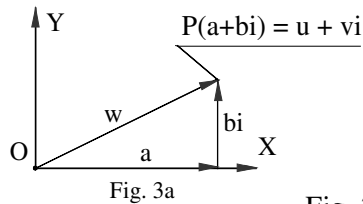

Fig. 3

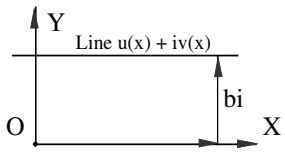

Fig. 3b

If we substitute $\mathrm{w}=\mathrm{a}+\mathrm{bi}=\mathrm{x}+\mathrm{yi}$ in the polynomial and give $b$ a numerical value, but leave $x$ variable, then obviously remain just the terms with $\mathrm{x}$ unknown. These have the powers 0 to $\mathrm{n}$. All these terms form than two polynomials in $x$, one multiplied by $i$ (imaginary part), and another without $i$ (real part). They correspond to the line at distance $b$ (b can be chosen numerically) one being the real, the other the imaginary part). If these polynomials are solved, they give $n$ points for the root lines of the real part and $n$ for the imaginary part. (See Fig. $3 b$ )

Only the real roots have to be calculated, the complex roots are discarded being outside of the line at distance $b$.

\section{K. Different possible positions of the polynomial centre.}

The polynomial centre was defined in the first part of this study (in 2002) as the geometrical centre of all roots both in direction $x$ and y. (See [7] Par. (2), Pg. 841)

In case of a single multiple root (i.e. there are no other roots than the multiple root) all root lines are straight lines which intersect where the multiple root is. There then both the real and imaginary part reduce to zero (so this is a complex root) and the polynomial center is also there (all roots are concentated in one point).

So in this case all root lines pass exactly through the polynomial center and they are straight lines and coincide with the asymptotes.

In case of circular (binary) polynomials in the form $\mathrm{w}^{\mathrm{n}}-1$ the polynomial centre coincides with the center of the circle on which the roots are, this being the best approximation for the action of all roots.

More about such polynomials see [7], Par 5A2., Pg 843. 
For other polynomials the asymptotes correspond to $\mathrm{w}^{\mathrm{n}}$ because the other terms for high values of $w$ are negligible against $\mathrm{w}^{\mathrm{n}}$ and therefore this case = especially for points far from the center - the polynimial center is similar to (i.e. the best approximation for) the case of a multiple root. It is also an invariant of the polynomial and depends on all roots. All practical cases which I studied confirmed this hypothesis.

\section{Solving polynomials}

To solve polynomials even with complex roots is simple if there is an adequate method and computer program.

Such a method is Newton's method in the complex domain. In order to see how this method works, let's see first Newton's method in the real domain.

According to the real domain method, for a given $\mathrm{x}$ value, the polynomial's value $\mathrm{z}=\mathrm{P}(\mathrm{x})$ and the value of its derivative is $\mathrm{z}^{\prime}=\frac{d P(x)}{d x}=\frac{d z}{d x}$ are known (see Fig. 4).

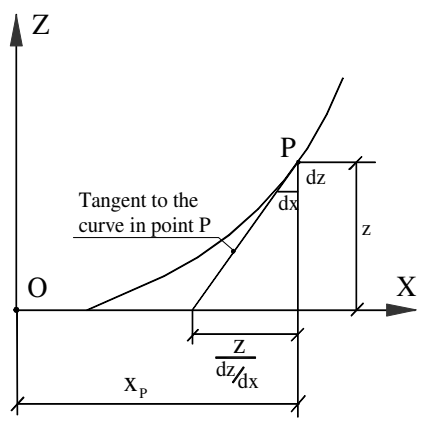

Fig. 4

But $z^{\prime}$ is the trigonometric tangent of the geometrical tangent to the polynomial's curve. Then the point where the geometrical tangent crosses the Ox axis will be at $\mathrm{x}-\frac{z}{z^{\prime}}$. This is now an approximation closer to a root of the polynomial. In many manuals $\frac{z}{z^{\prime}}$ is noted with $\frac{f}{f^{\prime}}$ (function $\mathrm{f}$ and its derivative $\mathrm{f}^{\prime}$ ).

In the complex domain, the function with a complex variable is

$$
\begin{aligned}
& \mathrm{f}=\mathrm{u}+\mathrm{vi} \text { and its derivative is } \\
& \mathrm{f}=\mathrm{u}+\mathrm{v} \text { ' } \mathrm{i} \text { where } \mathrm{f} \text { '. } \mathrm{u} \text { ' and } \mathrm{v} \text { ' are derivatives of } \mathrm{f} \text {, } \\
& \text { both with respect to } \mathrm{x} \text {. }
\end{aligned}
$$

If we divide $f$ with $f$ ' according to the rule of division of complex numbers we obtain

$$
\frac{f}{f^{\prime}}=\frac{u+v i}{u^{\prime}+v^{\prime} i}=\frac{u u^{\prime}+v v^{\prime}}{u^{\prime 2}+v^{\prime 2}}+\frac{v u^{\prime}-u v^{\prime}}{u^{\prime 2}+v^{\prime 2}} i
$$

The obtained two fractions subtracted from an initial value of an arbitrary complex number $\mathrm{w}=\mathrm{x}+\mathrm{yi}$ give a new approximation of $\mathrm{w}$. This is than repeated until value of $\frac{f}{f^{\prime}}$ becomes negligible.

J.B. Moore in [1] doesn't mention the name of Newton. He calls the above approximations, i.e. the value of $\frac{f}{f^{\prime}}$ 'steepest descent vector' ( $\mathrm{s}$ d v) but arrives to the same expression as given above.

For the s.d.v. see also [7] Par. 6A., Pg. 845

The above relation inserted in a computer program gives after a few steps value of one root. Then a synthetic division has to be performed, that means the polynomial is divided by $\mathrm{w}-\mathrm{w}_{1}$ where $\mathrm{w}_{1}$ is the value of the calculated root. After this the polynomial's degree reduces by 1 .

When $\mathrm{n}=1$, the root's value is calculated directly.

Observations regarding this method.

1.) The program works well also for polynomials with real coefficients or real roots, but then the imaginary part of the coefficients has to be set to zero. 2.) Whereas Newton's method in the real domain works only if the initial guess is close to a root, there is no such restriction in case of the program in complex domain. 3.) If the program enters in an 'endless loop' (it happens extraordinarily seldom), then increase initial guess, or change sign of the imaginary part (i.e. change the initial guess $1+\mathrm{i}$ to $10+10 \mathrm{i}, 100+100 \mathrm{i}, 1$ $-\mathrm{i}, 10-10 \mathrm{i}, 100-100 \mathrm{i}$ etc)

Note.

The size of this paper doesn't permit to give a complete listing of this or other programs. If you are interested in more details, please write to my e-mail address.

M. Rotation of the asymptotes of a multiple root multiplied by a complex constant.

A polynomial which has only one $n$ times multiple root, i.e.

$$
\begin{aligned}
& P_{n}=w^{n}=(x+b i)^{n} \quad \text { or in the trigonometric form: } \\
& P_{n}=\rho^{n}(\cos n \varphi+i \operatorname{sinn} \varphi)
\end{aligned}
$$

If this expression is multiplied by a complex number in the form $\mathrm{a}+\mathrm{bi}=\mathrm{a}(1+\mathrm{ki}) \quad$ where $\mathrm{k}=\frac{b}{a}=\tan \alpha \quad \alpha=\tan ^{-1}(\mathrm{k})$ (using for the argument of the complex multiplier $1+$ ki the letter $\alpha$ instead of $\varphi$ in order to make it different of $\varphi$ of the multiple root) then results

$$
(1+k i) w^{n}=\cos n \varphi-k \sin n \varphi+[\sin n \varphi+k \cos n \varphi] i
$$

Equating the real part and the imaginary part separately with zero we obtain

$$
\begin{aligned}
& \tan \mathrm{n} \varphi=\frac{1}{k}=\tan \left(90^{\circ}-\alpha\right) \quad \text { or } \mathrm{n} \varphi=90^{\circ}-\alpha \text { or } \\
& \varphi_{\mathrm{r}}=\frac{90^{\circ}-\alpha}{n}+\mathrm{k}_{1} \frac{\pi}{n}
\end{aligned}
$$


and similarly for the imaginary part

$$
\begin{aligned}
& \tan \mathrm{n} \varphi=-\mathrm{k}=-\tan \alpha \text { or } \mathrm{n} \varphi=-\alpha \text { or } \\
& \varphi_{\mathrm{i}}=\frac{-\alpha}{n}+\mathrm{k}_{1} \frac{\pi}{n}
\end{aligned}
$$

where $\mathrm{k}_{1}=1,2, \ldots \mathrm{n}-1 \quad$ (all integer numbers).

Relations (3a) and (3b) show directions of the lines along which the real or imaginary part of $\mathrm{w}^{\mathrm{n}}$ is equal with zero, i.e. their root lines.

$\varphi_{\mathrm{r}}$ and $\varphi_{\mathrm{i}}$ are measured from the reference axis $\mathrm{Ox}$

The term $\mathrm{k}_{1} \frac{\pi}{n}$ in these relations is called the term of multiplicity, because it adds to $\varphi_{\mathrm{r}}$ or $\varphi_{\mathrm{i}} \mathrm{n}-1$ more directions. Proof:

If $\varphi_{\mathrm{r}}$ or $\varphi_{\mathrm{i}}$ is substituted in the expression of $\mathrm{Pn}=\mathrm{w}^{\mathrm{n}}$ then the arguments $\varphi_{\mathrm{r}}+\mathrm{k}_{1} \frac{\pi}{n}$ or $\varphi_{\mathrm{i}}+\mathrm{k}_{1} \frac{\pi}{n}$ become $\operatorname{n}_{\mathrm{r}}+\pi$ resp $n \varphi_{i}+\pi$, but if for $n \varphi_{r}$ or $n \varphi_{r} \quad P n=0$ then it will be zero also for $\varphi_{\mathrm{r}}+\pi \operatorname{resp} \varphi_{\mathrm{i}}+\pi$.

\section{N. Consequences of relation (3a) or (3b)}

Consider a polynomial which has a triple root in the origin and another root at $-3-2 \mathrm{i}$. (See Fig. 5). It can be written as

$$
P_{4}=x^{3}(x+3+2 i)
$$

In the origin $\mathrm{x}=0$ and the parenthesis expression reduces

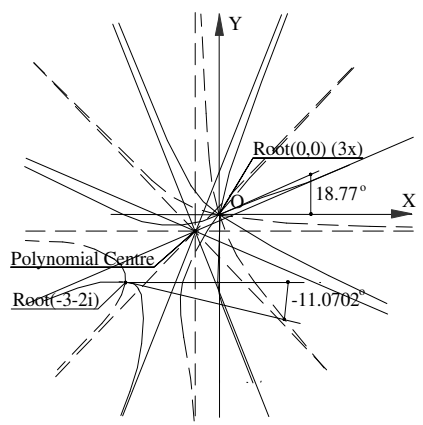

Fig. 5

to the complex constant $3+2 i=3\left(1+\frac{2}{3} i\right) \quad$ so in this case $\mathrm{k}=\frac{2}{3}=.6666 \quad \alpha=\tan ^{-1}\left(\frac{2}{3}\right)=33.690067^{\circ}$

This is exactly the case presented in the previous paragraph (M.), i.e. the complex multiple $\operatorname{root} \mathrm{x}^{3}\left(=\mathrm{w}^{3}\right)$ is multiplied by the complex constant $3+2 \mathrm{i}$.
Direction of the real root line will be then given by the angle $\varphi_{\mathrm{r}}$ equal with (using relations from Par. M.):

$$
\begin{aligned}
& \varphi_{\mathrm{r}}=\frac{90^{\circ}-\alpha}{n}+\mathrm{k}_{1} \frac{\pi}{3}=18.77^{\circ}+60^{\circ} \text { where } \\
& \alpha=\tan ^{-1}\left(\frac{2}{3}\right)=33.690077^{\circ} \mathrm{n}=3 \quad \text { (multiplicity of the }
\end{aligned}
$$

root) $\mathrm{k}_{1}=0,1,2$

Note that in these relations $n$ is the multiplicity, i.e. the number of roots in the multiple root (in this case $n=3$ ) and not the order of the polynomial (which in this case is 4 !)

Hence results that this polynomial has root lines which in the origin $\mathrm{O}$ (where in this case the triple root is) are rotated by $\varphi_{\mathrm{r}}$ and $\varphi_{\mathrm{i}}$ and to these directions are added two (in general n-1) more, so the total of directions in which the polynomials real or imaginary part is zero is is equal with $n$, i.e. the order of multiplicity (in general $\mathrm{n}$ and in this case 3) of the root.

The factor $3+2 \mathrm{i}$ is the influence of the other root on the multiple root. It causes a rotation of all root lines which pass through the multiple (in this case triple) root by $\varphi_{\mathrm{r}}$ resp $\varphi_{\mathrm{i}}$ but the interval between the lines remains always the same $\left(\frac{\pi}{3}\right)$ because of the term $\mathrm{k}_{1} \frac{\pi}{3}$ (in general $\mathrm{k} 1 \frac{\pi}{n}$ )

Further it is easy to see that the complex multiplier is $3+2 \mathrm{i}$ $=1+\frac{2}{3} \mathrm{i}=\mathrm{a}\left(1+\frac{b}{a}\right)$ and $\alpha=\tan ^{-1}\left(\frac{b}{a}\right) \quad$ where $\mathrm{a}$ is the real part and $b$ the imaginary part of the simple root In this concrete case (see Fig. 5) it was found that $\varphi_{\mathrm{r}}=$ $18.77^{\circ}$

This is the angle of the tangent to the first real root line with the Ox axis. The other root lines are at equal intervals of $\frac{\pi}{3}$

\section{O. Case of several multipliers.}

In case that the polynomial has more than one complex roots in different points, these can be multiplied as complex numbers and their product is

$$
\rho_{1} \rho_{2} \rho_{3} \ldots\left[\cos \left(\alpha_{1}+\alpha_{2}+\ldots\right)+i \sin \left(\alpha_{1}+\alpha_{2}+\ldots\right)\right]
$$

The arguments of the roots can be added up to

$$
\alpha_{\mathrm{tot}}=\tan ^{-1}\left(\mathrm{k}_{1}\right)+\tan ^{-1}\left(\mathrm{k}_{2}\right) \ldots
$$

Each argument is measured from the positive Ox axis. In case of a Root Number $\mathrm{k}$, best method is to set this root at the origin, but so that the relative position (i.e. distance) between the roots remains the same. (The simplest solution is then to move the reference axes and not the roots!). All roots have to enter in the sum except the Root $\mathrm{k}$ 
If there is a multiple root, it is like several simple roots added up, i.e. the argument of the multiple root is multiplied by the number of multiplicity of the root.

The total argument $\alpha_{\text {tot }}$ is then used in the relations of Par. M. (instead of $\alpha$ ).

This theory shows that the root lines at each root of a polyno-mial are rotated by a value which depends on all other roots of the polynomial. This has some similarity with a system of celestial bodies (like the sun and its planets) where each planet influences the others, but in case of a polynomial the influence depends only on the angle of the root, relative to the other root. The angles of all roots are then added up numerically.

\section{P. Rotation of the root lines at a simple root.}

With the same relation as in Par. M. to O. we can calculate also the rotation of the root lines at the simple root $3+2 \mathrm{i}$. The 3 times multiple root is equivalent with three simple roots. (See also observations in the previous paragraph O. regarding multiple multipliers).

For one root we have

$$
\begin{gathered}
\alpha=\tan ^{-1}\left(\frac{2}{3}\right)=33.69^{\circ} \quad \text { For three roots: } \\
\alpha_{\mathrm{tot}}=3 \cdot \tan ^{-1}\left(\frac{2}{3}\right)=3 \cdot 33.69=101.07^{\circ} \\
\text { Then } \varphi_{\mathrm{r}}=\frac{90^{\circ}-\alpha_{t o t}}{n}=\frac{90-101.07}{1}=-11.07^{\circ}
\end{gathered}
$$

See for this again Fig. 5.

\section{Q. Polynomials multiplied by a complex number.}

The first coefficient (i.e. that of $x^{n}$ ) is in most cases of polynomials equal with 1 and $C_{n y}=0$ (i.e. $C_{n}$ has no imaginary part). If a polynomial is given in form (2) and the multiplications are performed to obtain form (1), then $C_{n}$ will result $=1$ (real), even if all roots are complex.

So if $\mathrm{C}_{\mathrm{ny}} \neq 0$ then it can be assumed that the polynomial was multiplied by $\mathrm{C}_{\mathrm{nx}}+\mathrm{i} \mathrm{C}_{\mathrm{ny}}$. This complex factor then produces a rotation of the asymptotes which can be calculated with Rel. (3) where in this case $\mathrm{k}=\frac{C_{n y}}{C_{n x}} \quad \alpha=$ $\tan ^{-1}(\mathrm{k})$

Or, another (better) method is to divide the whole polynomial by the first, complex coefficient $\mathrm{C}_{\mathrm{nx}}+\mathrm{i}_{\mathrm{ny}}$ After this division $\mathrm{C}_{\mathrm{nx}}=1 \quad \mathrm{C}_{\mathrm{ny}}=0$ and the polynomial will be a 'regular' polynomial.

The roots remain after division the same, because the polynomial is multiplied just by a constant.

The polynomial centre can be calculated from the divided polynomial. It is the same for both polynomials, because it is a function of all roots, and these are the same for both.

As an example consider the following polynomial, given in Ref [5]

$$
2 x^{4}-30 x^{3}+163 x^{2}-1773+\left(3 x^{4}+2 x^{3}+472 x+4208\right) i
$$

In this case $\mathrm{C}_{\mathrm{ny}}$ is not $0, \mathrm{k}=\frac{C_{n y}}{C_{n x}}=\frac{3}{2}=1.5$

$\alpha=\tan ^{-1}(1.5)=$ therefore all asymptotes are rotated

by

$\varphi_{\mathrm{r}}=\frac{90^{\circ}-\tan ^{-1}(1.5)}{4}=8.422517^{\circ}$

$\varphi_{\mathrm{r}}$ is measured from the Ox axis

If the whole polynomial (i.e. all coefficients) are divided by the first coefficient, i.e. $\mathrm{C}_{\mathrm{x} 4}+\mathrm{C}_{\mathrm{y} 4} \mathrm{i}=2+3 \mathrm{i}$ then we obtain the following polynomial:

$$
\begin{aligned}
& 13 \mathrm{x}^{4}-54 \mathrm{x}^{3}+326 \mathrm{x}^{2}+1416 \mathrm{x}+9078+\left(94 \mathrm{x}^{3}-489 \mathrm{x}^{2}+\right. \\
& 944 \mathrm{x}+13735) \mathrm{i} \text { or } \\
& \mathrm{x}^{4}-4.15384815 \mathrm{x}^{3}+25.076923 \mathrm{x}^{2}+108.923077 \mathrm{x} \\
& 698.307692+\left(7.230769 \mathrm{x}^{3}-37.615385 \mathrm{x}^{2}+\right. \\
& 72.615385 \mathrm{x}+1056.5385) \mathrm{i}
\end{aligned}
$$

This polynomial has the asymptotess according to the general rule (first real asymptote at $\frac{\pi}{2 n}$ ) and the same roots. Hence results the polynomial centre as:

$$
\begin{aligned}
& \operatorname{PolCtr}_{\mathrm{x}}=-\frac{C_{x}(n-1)}{n C x(n)}=\frac{54}{13 \cdot 4}=1.0384615 \\
& \text { PolCtr }_{\mathrm{y}}=-\frac{C_{y}(n-1)}{n C x(n)}=-\frac{94}{13 \cdot 4}=-1.8076923
\end{aligned}
$$

\section{R. Sum of the angles of the roots of a polynomial related to a point $M$ on a root line of the real part.}

If the sum of the angles of all roots of a polynomial related to a point $\mathrm{M}$ is equal with $90^{\circ}$ then that points is situated on a root line of the real part.

\section{S. Sum of the angles of the roots of a polynomial related to a point $M$ on a root line of the imaginary part.}

If the sum of the angles of all roots of a polynomial related to a Point $\mathrm{M}$ is equal with 0 then the point is situated on a root line of the imaginary part.

\section{T. Proof of Par. R and S.}

If the point $\mathrm{M}$ is placed in the origin then the value of the polynomial in point $\mathrm{M}$ is equal with

$$
\mathrm{P}=\left(-\mathrm{a}_{1}-\mathrm{b} 1 \mathrm{i}\right)\left(-\mathrm{a}_{2}-\mathrm{b}_{2} \mathrm{i}\right) \quad\left(-\mathrm{a}_{\mathrm{n}}-\mathrm{b}_{\mathrm{n}} \mathrm{i}\right)
$$

or written in trigonometric form

$$
P=\rho_{1} \rho_{2} \rho_{3} \ldots\left[\cos \left(\varphi_{1}+\varphi_{2}+\ldots\right)+\operatorname{isin}\left(\varphi_{1}+\varphi_{2}+\ldots\right)\right]
$$


From this expression it is obvious, that if the sum of the angles $\varphi_{1}, \varphi_{2} \ldots \varphi_{\mathrm{n}}$ is equal with $90^{\circ}$ then the real part of the polynomial is zero and if the sum is 0 then its imaginary part is zero (in point $\mathrm{M}$ ).

\section{U. Consequence of Paragraph $P$}

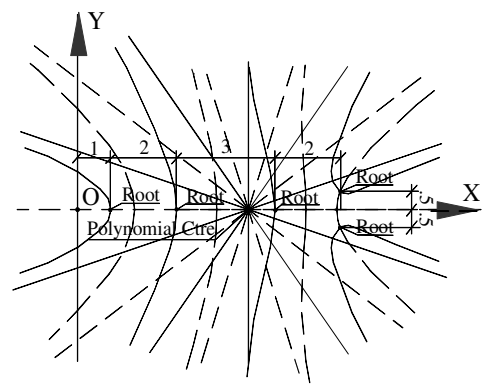

a. The original roots on the Ox axis

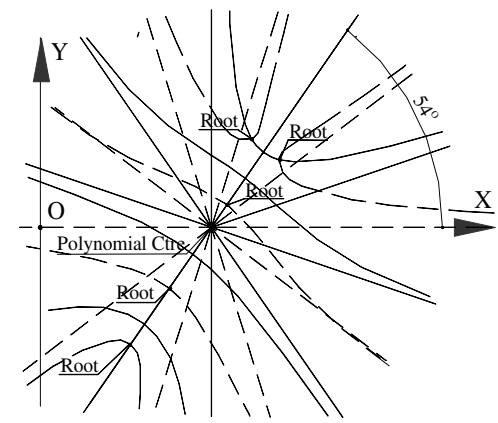

b. The roots rotated by 54

Fig. 6

If all roots of a polynomial are rotated by an angle $\varphi$ as a rigid body around the polynomial centre, then if $\varphi$ points in a principal direction then all root lines of the polynomial rotate by the angle $\varphi$ but their status could be reversed (i.e. a root line which was of the real part becomes of the imaginary part and vice-versa) if the direction defined by $\varphi$ corresponds to another principal direction.

Proof.

Because of the rigid rotation, the angle at which a root is seen remains the same as before, but the angle $\varphi$ is added to it $n$ times (for each root). This additional value of $n \varphi$ may cause that the total angle to be a multiple of $90^{\circ}$. In case of Fig. 6 the rotation being $54^{\circ}, \mathrm{n} \varphi=5 \cdot 54=270=180^{\circ}+90^{\circ}$. The $180^{\circ}$ doesn't change anything, but the $90^{\circ}$ changes. If sum of the angles initially was 0 , now it becomes $90^{\circ}$, and if it was $90^{\circ}$, now it will be $180^{\circ}$, that means all root lines will change their status.

As an example consider a polynomial with real roots at $\mathrm{x}=$ $1, x=3$ and $x=6$ and a pair of complex roots $x=8+.5 \mathrm{i}$ and $8-.5 \mathrm{i}$. This polynomial has the equation

$$
\begin{aligned}
& x^{5}-25 x^{4}+225 x^{3}-903 x^{2}+1566 x-864 \\
& +(-6 x 4+106 x 3-622 x 2+1350 x-828) i
\end{aligned}
$$

If all roots are rotated by $54^{\circ}$ around the polynomial centre (at $x=5.2$ ) in positive direction then the roots will be:

$\begin{array}{lr}2.7313019-3.397871 \mathrm{i} & 3.906872445- \\ 1.779837386 \mathrm{i} & \\ 5.6702282+.647213595 \mathrm{i} & 6.4412902 \\ +2.55914921 \mathrm{i} & \\ 7.250307204+1.971354968 \mathrm{i} & \end{array}$

These roots correspond to following polynomial (using Rel. [2])

$$
\begin{aligned}
& x^{5}-26 x^{4}+276.3176748 x^{3}-1572.5094075 x^{2}+ \\
& 4222.1774568 x-4701.3827155856 \\
& +\left(-18.2127322 x^{3}+288.704436236 x^{2}\right. \\
& \quad-1569.03213486 x+2973.05628351) i
\end{aligned}
$$

This polynomial has the same root lines as the previous one but all root lines are reversed, i.e. if a root line was before real, now it will be imaginary and vice-versa.

See also the proof at the beginning of this paragraph.

Observe that the rotation of $54^{\circ}$ is an integer multiple of

$\frac{\pi}{2 n}=18$ which is the interval between two different root

lines (one of the real part and one of the imaginary part)

See Fig 6. for the initial and rotated polynomial

\section{References}

[1] Moore, J. B. A Convergent Algorithm for Solving Polynomial Equations, Journal of the Association for Computing Machinery, Vol. 14, No. 2, April 1967. pp. 211315

[2] Garrett Birkhoff, Saunders Mac Lane, A Survey of Modern Algebra, MacMillan Publishing Co, Inc, New York, 1977.

[3] A. Ralston, P. Rabinowitz, A First Course in Numerical Analysis, 2nd ed, McGraw-Hill, New York, 1978.

[4] F.R. Ruckdeschel, BASIC Scientific Subroutines, Vol I and II, Peterborough, NH: BYTE Books, 1981.

[5] W.H. Press, B. P. Flannery, S. A. Teukolsky, W. T. Vetterling, Numerical Recipes, The Art of Scientific Computing, Cambridge University Press, Cambridge, New York, New Rochelle, Melbourne, Sydney, 1986-7

[6] Osborne/MacGraw-Hill Science and Engineering Programs Apple II Edition

[7] The $27^{\text {th }}$ Annual Congress of the American Romanian /Academy of Arts and Sciences (ARA), May 29 - June 2 2002, Universitatea of Oradea, Proceedings, Vol. II, Pg. 841, Nicholas Schmidt, Theory of the Root Lines of the General Polynomial (Part 1) 